\title{
GAS-SOLID TRICKLE FLOW HYDRODYNAMICS IN A PACKED COLUMN
}

\author{
K. R. WESTERTERP and M. KUCZYNSKI \\ Chemical Reaction Engineering Laboratories, Department of Chemical Technology, Twente University of \\ Technology, P.O. Box 217, 7500 AE Enschede, The Netherlands
}

(Received 6 May 1986; accepted for publication 6 October 1986)

\begin{abstract}
The pressure gradient and the static and the dynamic hold-up have been measured for a system consisting of a Fluid Cracking Catalyst (FCC) of $30-150 \times 10^{-6} \mathrm{~m}$ diameter, trickling over a packed bed and with a gas streaming in countercurrent flow. The experiments were carried out at ambient conditions using a glass column of $25 \times 10^{-3}$ m diameter. The packing material consisted of $8 \times 8 \times 3 \mathrm{~mm}$ ceramic Raschig rings, a mixture of $7 \times 7 \times 1 \mathrm{~mm}$ glass Raschig rings and $5 \times 5 \mathrm{~mm}$ catalyst pellets and of stacked Kerapak ceramic mixing units of Sulzer, each unit $50 \mathrm{~mm}$ long and $25 \mathrm{~mm}$ in diameter. Four different gases have been tested. A correlation for the pressure gradient in the preloading region is derived based on the Ergun equation and taking into account the internal gas recirculation due to the solids trickles. The void fraction of the trickles is found to be independent of the physical properties of the gas phase. The bchaviour of the GSTF-system in the preloading regime and the phenomena of loading and flooding are discussed. A correlation is given which relates the boundary between preloading and loading with the particle and gas properties and the solids flow rate.
\end{abstract}

\section{INTRODUCTION}

In many processes, e.g. heat exchange, adsorption, drying or chemical reaction, a countercurrent contacting of gas and solids is required. To this end a large variety of equipment has been developed, such as moving beds, multistage fluid beds, spray columns, zigzag contactors or rotary furnaces. Mostly high mass and heat transfer rates and a low axial mixing in both phases is desired, whereas the pressure drop over the contactor determines the energy consumption for the contacting process. Gas-solid trickle flow (GSTF), which is a relatively new operation in process engineering, exhibits some very attractive properties: the investigators report on the low pressure drop, high mass and heat transfer rates and low axial mixing in both phases. Furthermore it exhibits a good operating stability and the construction of a GSTF-column is simple. For design purposes reliable criteria are necessary to predict, e.g. the pressure drop and the solids hold-up. Regretfully there is still an important lack of understanding of the hydrodynamic behaviour of the GSTF system. For the operating regime where freely falling solid trickles are observed, a strong similarity in hydrodynamic behaviour to the gas-liquid trickle flow is observed: existence of a static and a dynamic hold-up and the phenomena of loading and flooding. However, it must be borne in mind that in many aspects the nature of the GSTF differs strongly from that of the gas-liquid flow. So, for example, the static hold-up of a liquid in a bed of solid particles is caused by adhesion forces, while there are no equivalent forces for a solid-solid system. The solids trickles in between the particles contain gas, which to some extent will be carried away in the downward direction. In gas-liquid systems the trickles of liquid are not porous.

The present study has been executed as a part of our Gas-Solid-Solid Trickle Flow Reactor (GSSTFR)- project, for which we had to determine the hydrodynamically stable operating ranges of such a reactor. In this project two solid phases were present in the reactor: a fixed bed of catalyst particles with diameters in the order of magnitude of millimeters and a powder trickling downwards over these catalyst particles, with a particle size in the order of magnitude of 10-100 microns and to be used to adsorb a component out of the gas flowing upwards through the reactor. We used a glass apparatus as a "cold model" of our GSSTFR. The diameter of the column is equal to that of our high pressure GSSTFR. The catalyst for the methanol synthesis consists of $5 \times 5 \mathrm{~mm}$ cylindrical pellets. In a bed packed with such particles trickle flow of solids is hardly possible: clogging occurs very quickly. Therefore in this study we used a bed consisting of catalyst pellets diluted with $7 \times 7 \times 1 \mathrm{~mm}$ glass Raschig rings in order to increase the void fraction of the bed and to obtain a stable trickle flow. Two other packing materials also have been tested: $8 \times 8 \times 3 \mathrm{~mm}$ Raschig rings and Sulzer Kerapak mixing units of $25 \mathrm{~mm}$ diameter and $50 \mathrm{~mm}$ long. Both are made of an alumina catalyst carrier. We used the four gases air, argon, helium and Freon 12, so that densities and kinematic viscosities differed with a factor of 30 and 44, respectively. This should enable us to make predictions in an operating regime of higher pressures and temperatures.

\section{LITERATURE DATA}

Experiments on GSTF for sand and air flowing over a randomly packed column were reported upon by Claus, Vergnes and Le Goff (1976). From their pressure-drop measurements they observed three possible operating ranges for their system, which they called the "preloading region", the "loading zone" and 
the "flooding point", all these terms having the same significances as for a gas-liquid system. In the preloading zone they found the linear velocity of the solids to be independent of the solids feed flow. Furthermore, in the preloading zone the gas velocity had no influence on the solids hold-up or the linear velocity of the solids. Surprisingly the upwards flowing gas does not affect the solids particles in their falling motion due to the gravity, or also, possible drag forces are independent of the relative velocity between gas and solid. However, at the same time a very strong increase of the pressure drop is observed due to the flow of solids.

The study by Roes and van Swaaij (1978) supports these findings. Using a column filled with Pall rings, Raschig rings or cylindrical screens for the system airfluid cracking catalyst they observed a constant particle velocity in the "preloading region" and a constant particle slip velocity in the "loading zone". Further they observed that the pressure drop is mainly caused by the flow of solids and proposed an empirical correlation for the solids contribution to the pressure drop. Based on the constant values observed for the particle velocity $u_{\mathrm{p}}^{\circ}$ and the slip velocity $u_{\text {slip }}$, they also derived expressions for the dynamic hold-up, the loading point and the flooding point. Furthermore they concluded that data on gas-liquid systems can not be used to predict the behaviour of the GSTF system.

Large, Naud, Guigon and Bergougnou (1981) observed no true loading or flooding phenomena in their system of air and sand in a column filled with dumped Pall rings. At increasing gas loads they observed the formation of dead zones and separation of both phases in the vertical and also the radial direction. The particle velocity was found to be dependent on the gas velocity, also in the range of low gas flows.

Regretfully the literature data are not conclusive and a clear picture of the hydrodynamics of the gas-solid trickle flow cannot be obtained. We may conclude that the reported similarities between the GSTF and the gas-liquid trickle flow are only apparent.

\section{EXPERIMENTAL SET-UP AND PROCEDURES}

The experimental system is outlined in Fig. 1. The $0.8 \mathrm{~m}$ long glass column with a diameter of 25 $\times 10^{-3} \mathrm{~m}$ is equipped with a grid supporting the packing material. The height of the packing was around $0.7 \mathrm{~m}$; data of the packings used are given in Table 1. The column is fed at the bottom with gas: the flow can be adjusted with precision valves and using calibrated rotameters. The solids particles in the fluid bed below the column are transported pneumatically through a riser to the top of the column, where in a cyclone they are separated from the transport gas, the powder then drops into the column. The solids feed flow can be adjusted by changing the flow rate of the transport gas. The solids stream leaving the column at the bottom flows to the fluid bed. In order to prevent short-circuiting of gases a gas-lock is installed in the pipe connecting the bottom of the column to the fluid bed. For the determination of the pressure drop a

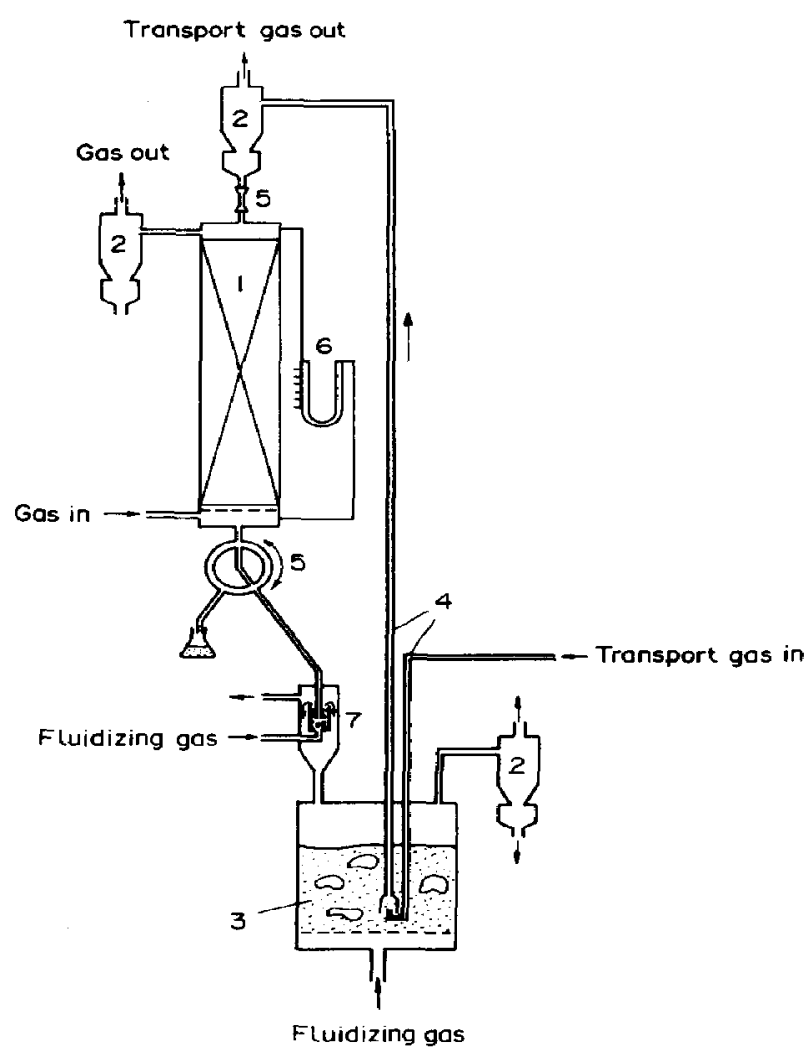

Fig. 1. The experimental setup. (1) glass column; (2) cyclone; (3) fluid bed; (4) solids riser; (5) valves; (6) micromanometer; (7) gas lock.

Table 1. The packing materials used

\begin{tabular}{|c|c|c|c|c|}
\hline & Packing & $\begin{array}{l}\text { dimensions } \\
\text { (mm) }\end{array}$ & $\underset{\left(m^{-1}\right)}{S_{y^{1}}}$ & $\varepsilon_{p}$ \\
\hline (1) & $\begin{array}{l}\text { Diluted catalyst: } \\
\text { glass Raschig rings } \\
+ \\
\text { catalyst pellets }{ }^{\dagger}\end{array}$ & $\begin{array}{l}7 \times 7 \times 1 \\
5 \times 5\end{array}$ & 680 & 0.58 \\
\hline (2) & $\begin{array}{l}\text { Raschig rings: } \\
\text { ceramic rings }\end{array}$ & $8 \times 8 \times 3$ & 536 & 0.45 \\
\hline (3) & $\begin{array}{l}\text { Kerapak: } \\
\text { Sulzer Kerapak } \\
\text { elements }\end{array}$ & $\begin{array}{l}\phi=25 \\
L=50\end{array}$ & \pm 1000 & 0.75 \\
\hline
\end{tabular}

${ }^{\dagger}$ Ratio of the Raschig rings to the catalyst pellets equals 1 to 2 by number of particles.

micromanometer is connected to both ends of the column. The dynamic hold-up can be measured by closing the ball valve above and switching the threeway valve below the column: the solids supply is interrupted whereas the solids still leaving the packed bed are collected and weighed. The static hold-up is determined by discharging the contents of the column, sieving and weighing the solid powder.

For the Raschig rings we used argon and air and for the Kerapak of Table 1 the experiments were carried 
out with air only. For the diluted catalyst packing also argon, helium and Freon 12 were used. The properties of the gases used are given in Table 2. The properties of the powder are specified in Table 3.

\section{RESULTS}

\section{Pressure gradient}

In Fig. 2 the pressure drop per unit length of the column for the system FCC-air is plotted versus the gas flow at constant solids flow rates. For all three packing materials, especially in the region of low gas flows the pressure gradient is strongly increased due to the solids flow. Without solids flow the pressure gradient over the Kerapak is lower by a factor of 3 to 10 than that over diluted catalyst or the Raschig rings. With flow of solids the situation is similar: the Kerapak packing exhibits by far the lowest pressure gradient. At equal gas and solids flow rates the pressure drop over the Raschig rings is remarkably lower than over the diluted catalyst. At incrcasing gas flow rates the slope of the curves increases; at high gas velocities this slope becomes even greater than that of the line for zero solids flow. A similar behaviour has been observed for the diluted catalyst packing using Freon 12 and helium, as can be seen in Fig. 3. At higher gas velncities, prior to the flooding an entrainment of the solids starts.

Also at zero gas flow a pressure gradient has been
Table 3. The properties of the adsorbent used

\begin{tabular}{lc}
\hline Composition & $83 \mathrm{wt} \% \mathrm{SiO}_{2}+13 \mathrm{wt} \%$ \\
& $\mathrm{Al}_{2} \mathrm{O}_{3}$ \\
Particle density & $813 \mathrm{~kg} / \mathrm{m}^{3}$ \\
Fixed bed density & $475 \mathrm{~kg} / \mathrm{m}^{3}$ \\
\hline
\end{tabular}

Particle diameter distribution (from sieve analysis)

\begin{tabular}{ccc}
$d_{\mathrm{p}} \times 10^{6}$ & $\begin{array}{c}\text { weight } \\
\text { fraction } \\
(\%)\end{array}$ & $\begin{array}{c}\text { cumulative } \\
\text { weight fraction } \\
(\%)\end{array}$ \\
\hline$<38$ & 0.1 & 0.1 \\
$38-45$ & 0.3 & 0.4 \\
$45-63$ & 6.3 & 6.7 \\
$63-90$ & 39.4 & 46.1 \\
$90-125$ & 41.3 & 87.4 \\
$125-150$ & 12.3 & 99.7 \\
$>150$ & 0.3 & 100 \\
\hline
\end{tabular}

measured, see Fig. 4. Its value increases with increasing solids feed flow. Of course, at both $G=0$ and $S=0$ the pressure drop is zero.

The dynamic hold-up $\beta_{\text {dyn }}$ consists of solids particles which continuously move through the packing so that after interrupting the solids flow they drain from the packing. The static hold-up $\beta_{\mathrm{st}}$ is retained in the bed and consists of particles which do not drain at zero solids flow. Both $\beta_{\text {dyn }}$ and $\beta_{s t}$ are defined as the respective volume fractions in the bed occupied by the solids

Table 2. The gases used and their properties at $p=0.101 \mathrm{MPa}$ and $T=293 \mathrm{~K}$

\begin{tabular}{lcccc}
\hline & & \multicolumn{2}{c}{ for our powder: } \\
gas & $\begin{array}{c}\rho_{\mathrm{g}} \\
\left(\mathrm{kg} \mathrm{m}^{-3}\right)\end{array}$ & $\begin{array}{c}v_{\mathrm{g}} \times 10^{6} \\
\left(\mathrm{~m}^{2} \mathrm{~s}^{-1}\right)\end{array}$ & $\begin{array}{c}d_{\mathrm{r}} \times 10^{6} \\
(\mathrm{~m})\end{array}$ & $\begin{array}{c}u_{\mathrm{r}} \\
\left(\mathrm{m} \mathrm{s}^{-1}\right)\end{array}$ \\
\hline argon & 1.67 & 13.2 & 32.4 & 0.38 \\
air & 1.20 & 14.6 & 33.6 & 0.46 \\
helium & 0.17 & 106 & 64.1 & 1.69 \\
Freon 12 & 5.1 & 2.4 & 15.3 & 0.16 \\
\hline
\end{tabular}

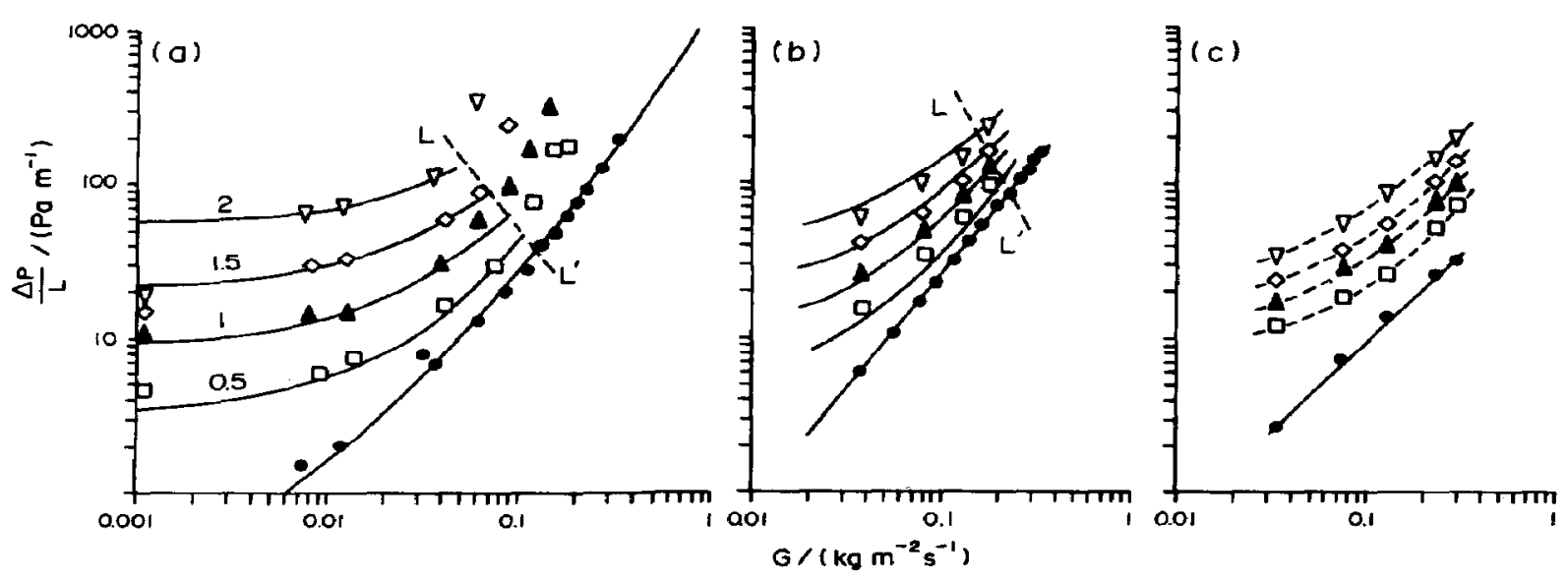

Fig. 2. The axial pressure gradient versus the flow rate of air; (a) diluted catalyst; (b) Raschig rings and (c) Kerapak. Solids flow rates $S\left(\mathrm{~kg} \mathrm{~m}^{-2} \mathrm{~s}^{-1}\right)$ are: $0 ; \square 0.5 ; A 1 ; 01.5 ; \nabla 2$. The solid lines are based on the modified Ergun equation. $\mathbf{L}-\mathbf{L}^{\prime}$-loading lines. 


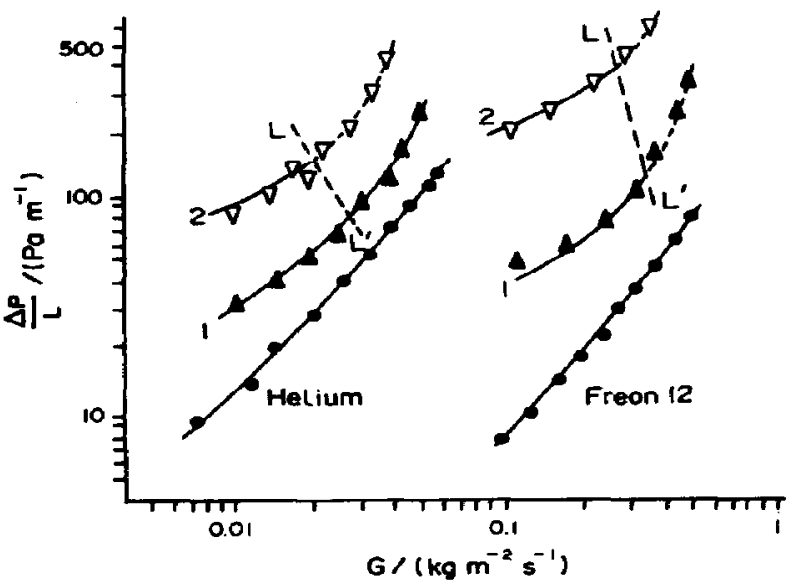

Fig. 3. The pressure gradient over the diluted catalyst packing measured using Freon 12 and helium. For symbols see Fig. 2.

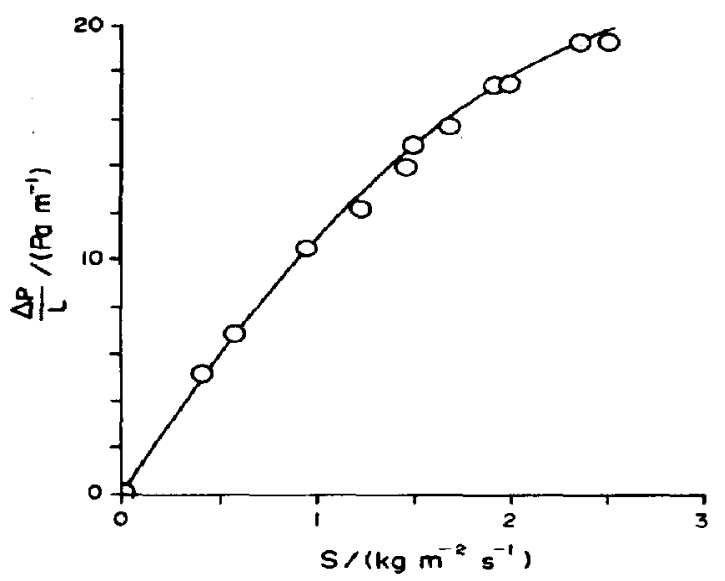

Fig. 4. Pressure gradient versus the solids flow rate at $G=0$.

particles and are calculated on the basis of the particle density of the solids. In Fig. 5 the static hold-up $\beta_{\text {st }}$ is plotted versus the gas flow rate for the three packings used. A slight increase of $\beta_{\mathrm{st}}$ is observed for increasing gas flow rates. The highest values of $\beta_{\text {st }}$ have been measured for the Raschig rings while for the Kerapak the static hold-up is by far the lowest.

In Fig. 6 plots of the dynamic hold-up $\beta_{\text {dyn }}$ versus the gas flow rate are shown. At low gas flows we observe for the diluted catalyst and for the Raschig rings that $\beta_{d y z}$ remains constant despite the increasing of the gas load. At increasing the gas flow even more the loading region is reached: in this region $\beta_{\mathrm{dyn}}$ does increase with increasing gas flow rate. In Fig. 6(a) and (b) we have estimated the operating points at which $\beta_{\mathrm{dyn}}$ starts to increase with increasing gas flow rates and have connected these points with the dotted "loading lines" L-L'. For the Raschig rings the loading starts at higher gas velocities than for the diluted catalyst. The Kerapak packing exhibits a different behaviour

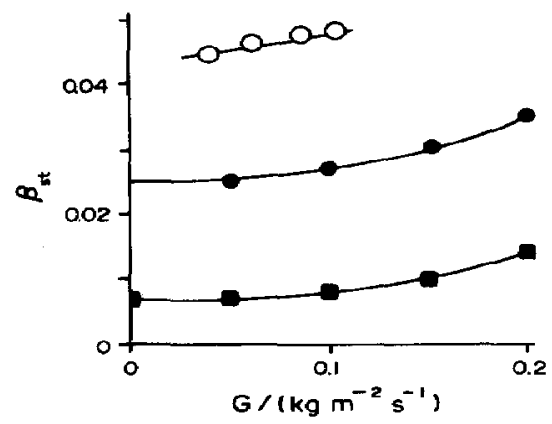

Fig. 5. The static holdup versus the gas flow rate: diluted catalyst; $O$ Raschig rings; Kerapak.

(Fig. 6c): despite the high void fraction and the low pressure drop, $\beta_{\mathrm{dyn}}$ starts to increase already at very low gas flow rates and no distinct loading point can be discerned.

The experimental data on the dynamic hold-up for the diluted catalyst and using helium, argon and Freon 12 give similar plots to those for air. For the loading range these data are given in Table 4. In this table, also the data in the loading regime for the system Raschig rings-air are given. In the preloading zone $\beta_{\mathrm{dyn}}$ appears to be independent of the physical properties of the gas phase. The loading lines as estimated from the $\beta_{\mathrm{dyn}}-G$ plots for the four gases used are shown in Fig. 7. The gas flow rate at the loading point decreases at an increasing solids flow rate.

To illustrate the influence of the solids flow rate on the dynamic hold-up, in Fig. $8 \beta_{d y n}$ is plotted vs $S$ for the diluted catalyst at three different gas flow rates. At zero gas flow the maximum solids flow rate is about $3.5 \mathrm{~kg} \mathrm{~m}^{-2} \mathrm{~s}^{-1}$; at $G=0.15 \mathrm{~kg} \mathrm{~m}^{-2} \mathrm{~s}^{-1}$ it is about $1 \mathrm{~kg} \mathrm{~m}^{-2} \mathrm{~s}^{-1}$. In the preloading zone the dynamic hold-up is proportional to the solids flow rate and independent of the gas flow rate. A similar dependence has been observed for the Raschig rings, whereas the Sulzer packing behaves differently, see Fig. 9. For this packing $\beta_{\mathrm{dyn}}$ depends on the gas flow rate in the entire range tested.

For the diluted catalyst packing we have determined the upper limits of the stable GSTF-operation called the flooding lines, using the four different gases. The results are shown in Fig. 10. Here the gas flow rates, at which either the clogging or the "blow-off" starts, are plotted versus the solids feed flow. The accuracy of the determination of the operating points where the flooding begins is low. We therefore plot the dotted "flooding ranges" in this figure. It can be seen that at higher solids flow rates the flooding occurs at lower gas flow rates.

For the dumped packings we have made the following qualitative observations. In the preloading region the solids flows in the form of trickles only. This can be seen from plate 1: here the white powder trickles over the diluted catalyst packing. At the loading point we observe that the trickles become unstable and that a fraction of the particles starts to move outside and 

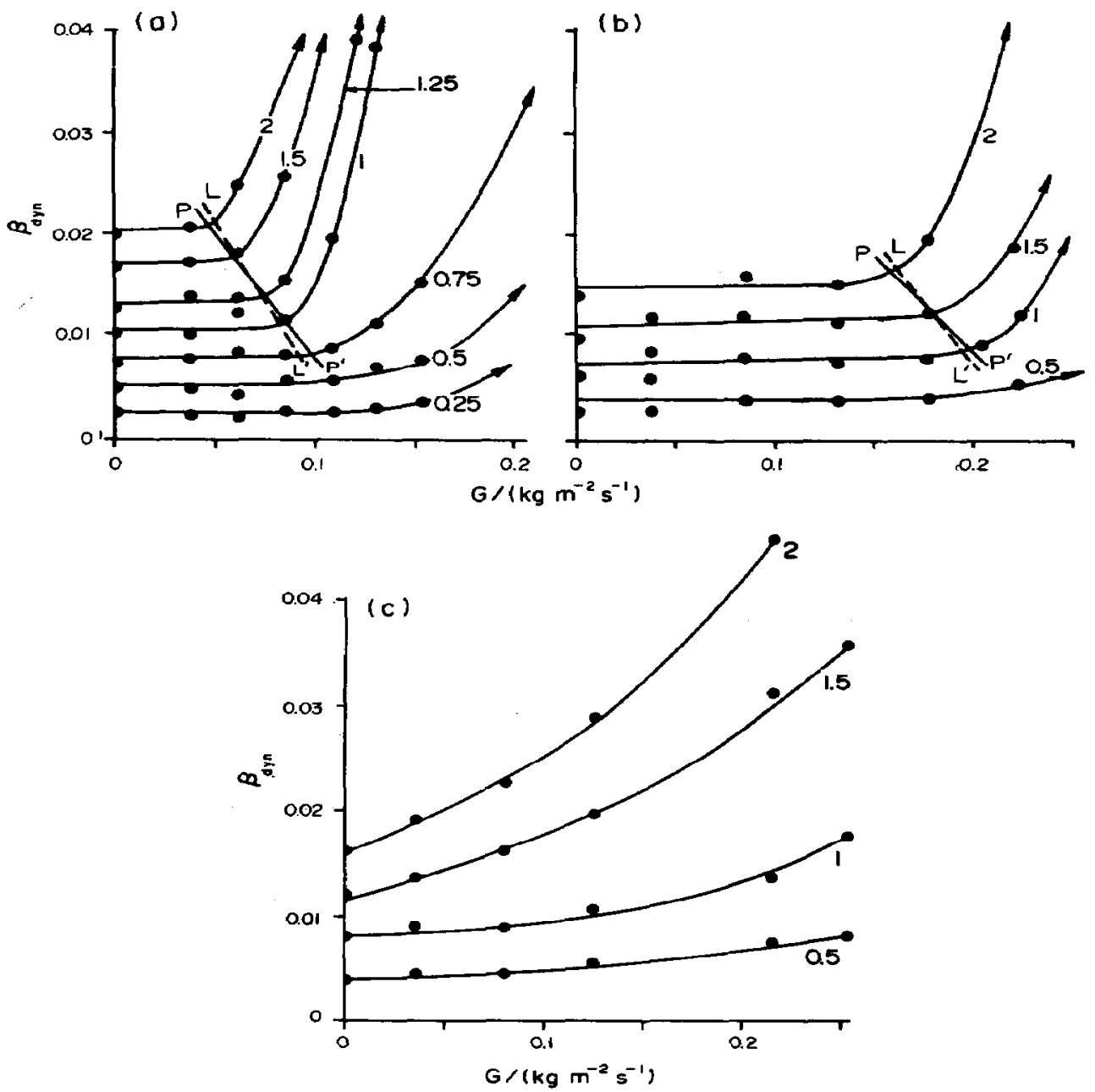

Fig. 6. The dynamic holdup versus the gas flow rate. The gas phase is air. (a) Diluted catalyst; (b) Raschig rings; (c) Kerapak. I -I:' loading lines; P-P' loading lines as predicted by eq. (6).

Table 4. The experimental data on loading and flooding for the diluted catalyst and the Raschig rings and the values of $u_{\text {slip }}$ calculated using eq. (12)

Gas $\quad \begin{gathered}S \\ \left(\mathrm{~kg} \mathrm{~m}^{-2} \mathrm{~s}^{-1}\right)\left(\mathrm{kg} \mathrm{m}^{-2} \mathrm{~s}^{-1}\right)\end{gathered} \beta_{\mathrm{dyn}} \quad \begin{gathered}u_{\mathrm{slip}} \\ (\mathrm{m} / \mathrm{s})\end{gathered}$

Diluted catalyst

air

$\begin{array}{llll}0.5 & 0.25 & 0.05 & 0.45^{\dagger} \\ 0.75 & 0.13 & 0.011 & 0.28 \\ & 0.15 & 0.015 & 0.30 \\ & 0.1 & 0.015 & 0.24 \\ & 0.125 & 0.03 & 0.25 \\ 1.25 & 0.19 & 0.06 & 0.30^{\dagger} \\ & 0.075 & 0.014 & 0.23 \\ & 0.1 & 0.022 & 0.23 \\ 1.5 & 0.125 & 0.039 & 0.25 \\ & 0.15 & 0.07 & 0.29^{\dagger} \\ 2 & 0.075 & 0.021 & 0.21 \\ & 0.12 & 0.07 & 0.24^{\dagger} \\ & 0.05 & 0.021 & 0.2 \\ & 0.075 & 0.03 & 0.21 \\ & 0.1 & 0.075 & 0.222^{\dagger}\end{array}$


Table 4. (Contd.)

\begin{tabular}{|c|c|c|c|c|}
\hline Gas & $\left(\mathrm{kg} \mathrm{m}^{-2}\right.$ & $\stackrel{G}{\left.m^{-2} s^{-1}\right)}$ & $\beta_{\mathrm{dyn}}$ & $\begin{array}{l}u_{\text {slip }} \\
(\mathrm{m} / \mathrm{s})\end{array}$ \\
\hline \multicolumn{5}{|l|}{ argon } \\
\hline & \multirow[t]{4}{*}{0.5} & 0.13 & 0.005 & 0.27 \\
\hline & & 0.16 & 0.007 & 0.27 \\
\hline & & 0.18 & 0.009 & 0.28 \\
\hline & & 0.34 & 0.04 & $0.43^{\dagger}$ \\
\hline & \multirow[t]{4}{*}{1} & 0.11 & 0.011 & 0.24 \\
\hline & & 0.15 & 0.012 & 0.27 \\
\hline & & 0.18 & 0.014 & 0.29 \\
\hline & & 0.29 & 0.045 & $0.39^{\dagger}$ \\
\hline & \multirow[t]{4}{*}{1.5} & 0.12 & 0.018 & 0.24 \\
\hline & & 0.14 & 0.021 & 0.26 \\
\hline & & 0.17 & 0.025 & 0.28 \\
\hline & & 0.24 & 0.045 & $0.34^{\dagger}$ \\
\hline & \multirow[t]{3}{*}{2} & 0.11 & 0.024 & 0.24 \\
\hline & & 0.15 & 0.030 & 0.26 \\
\hline & & 0.17 & 0.045 & $0.27^{+}$ \\
\hline \multicolumn{5}{|l|}{ helium } \\
\hline & \multirow[t]{4}{*}{0.5} & 0.031 & 0.007 & 0.45 \\
\hline & & 0.037 & 0.008 & 0.49 \\
\hline & & 0.045 & 0.014 & 0.56 \\
\hline & & 0.060 & 0.04 & $0.74^{\dagger}$ \\
\hline & \multirow[t]{4}{*}{1} & 0.024 & 0.011 & 0.38 \\
\hline & & 0.030 & 0.013 & 0.43 \\
\hline & & 0.039 & 0.02 & 0.51 \\
\hline & & 0.055 & 0.04 & $0.69^{\dagger}$ \\
\hline & \multirow[t]{4}{*}{1.5} & 0.023 & 0.015 & 0.39 \\
\hline & & 0.029 & 0.019 & 0.43 \\
\hline & & 0.036 & 0.024 & 0.5 \\
\hline & & 0.045 & 0.045 & $0.60^{\dagger}$ \\
\hline & \multirow[t]{4}{*}{2} & 0.022 & 0.021 & 0.37 \\
\hline & & 0.028 & 0.025 & 0.42 \\
\hline & & 0.030 & 0.033 & 0.43 \\
\hline & & 0.032 & 0.04 & $0.45^{\dagger}$ \\
\hline \multicolumn{5}{|l|}{ Freon 12} \\
\hline & \multirow[t]{3}{*}{0.5} & 0.46 & 0.021 & 0.27 \\
\hline & & 0.51 & 0.027 & 0.28 \\
\hline & & 0.54 & 0.07 & $0.24^{t}$ \\
\hline & \multirow[t]{4}{*}{1} & 0.40 & 0.011 & 0.26 \\
\hline & & 0.46 & 0.015 & 0.26 \\
\hline & & 0.51 & 0.018 & 0.26 \\
\hline & & 0.59 & 0.05 & $0.27^{\dagger}$ \\
\hline & \multirow[t]{5}{*}{1.5} & 0.32 & 0.016 & 0.24 \\
\hline & & 0.39 & 0.019 & 0.25 \\
\hline & & 0.44 & 0.026 & 0.24 \\
\hline & & 0.48 & 0.033 & 0.25 \\
\hline & & 0.50 & 0.07 & $0.24^{\dagger}$ \\
\hline & \multirow[t]{3}{*}{2} & 0.32 & 0.021 & 0.24 \\
\hline & & 0.36 & 0.027 & 0.23 \\
\hline & & 0.43 & 0.08 & $0.22^{\dagger}$ \\
\hline & \multicolumn{3}{|c|}{ Raschig rings } & \\
\hline \multirow{7}{*}{ air } & 0.5 & 0.225 & 0.005 & 0.65 \\
\hline & 1 & 0.225 & 0.012 & 0.54 \\
\hline & \multirow[t]{2}{*}{1.5} & 0.20 & 0.014 & 0.61 \\
\hline & & 0.225 & 0.019 & 0.60 \\
\hline & 2 & 0.18 & 0.019 & 0.56 \\
\hline & & 0.20 & 0.029 & 0.58 \\
\hline & & 0.225 & 0.041 & 0.64 \\
\hline
\end{tabular}

tFlooding.

independently of the trickles. At this point also the dynamic hold-up starts to increase, if either $G$ or $S$ or both are increased. At further increasing of $G$ the trickles remain unafected in their motion while the amount of the suspended particles increases gradually.
There was no recycling of the entrained solids at the top of our experimental column. Therefore instead of a sudden increase of the pressure drop and dynamic hold-up at flooding we observe an increased entrainment starting with the finest particles. This has also 


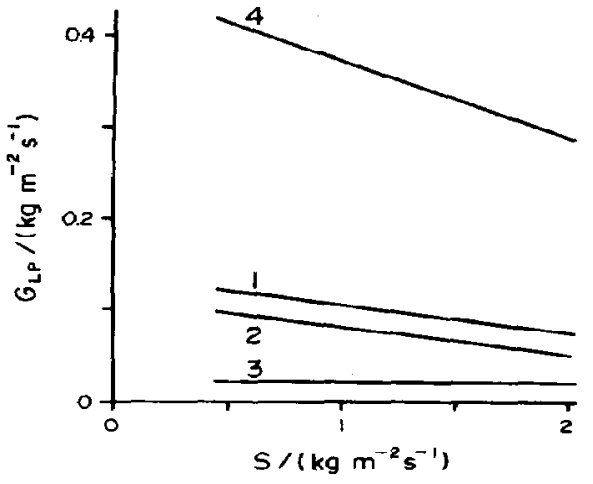

Fig. 7. The loading lines for the diluted catalyst measured for various gases: (1) argon; (2) air; (3) helium; (4) Freon 12.

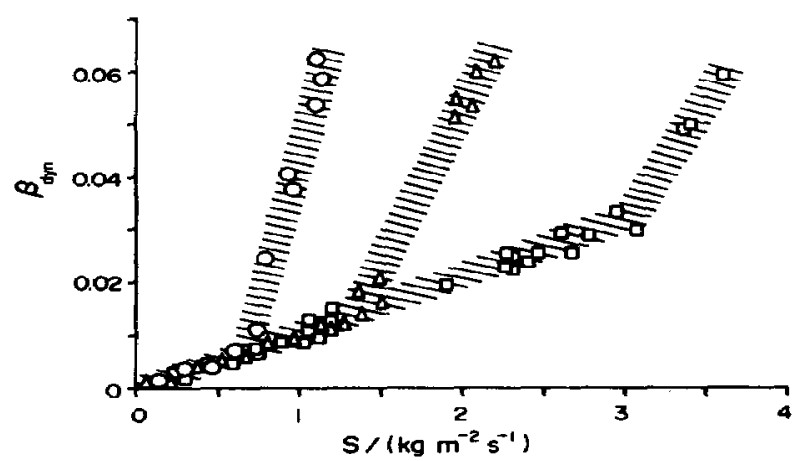

Fig. 8. The dynamic holdup in the diluted catalyst plotted versus the solids flow rate. The flow rates of the air $G\left(\mathrm{~kg} \mathrm{~m}^{-2} \mathrm{~s}^{-1}\right)$ are: $0 \quad 0 ; \Delta 0.09 ; 00.15$.

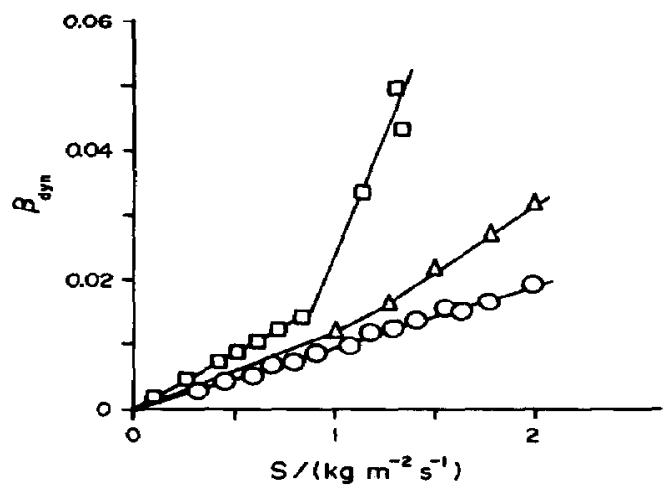

Fig. 9. The dynamic holdup versus the solids flow rate for the Kerapak. The flow rates of air $G\left(\mathrm{~kg} \mathrm{~m}^{-2} \mathrm{~s}^{-1}\right) ; 00, \Delta 0.15$; $\square 0.26$.

been described by van Swaaij and Verver (1986).

A visual observation of the inside of the Kerapak units was not possible.

\section{SOME REFLECTIONS ON THE PHENOMENA ON A MICROSCALE}

If we desire to derive correlations that describe the hydrodynamical behaviour of a GSSTFR it is a

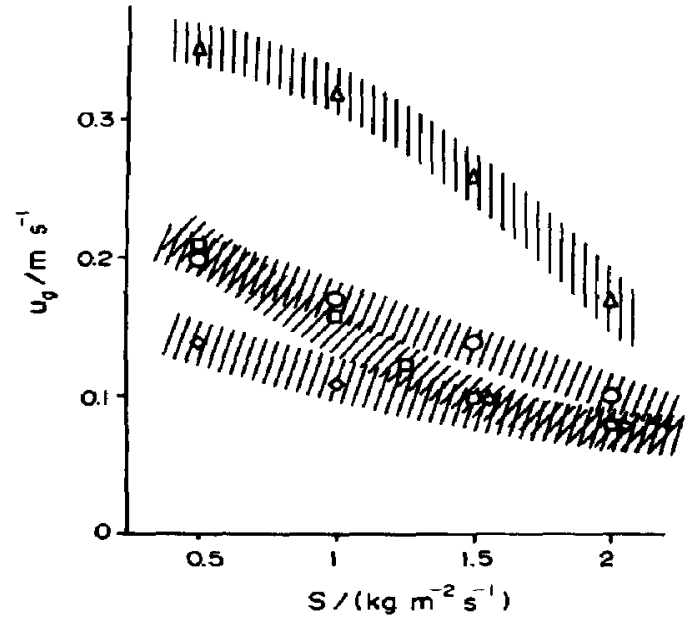

Fig. 10. The $u_{\mathrm{g}}$ vs $S$ plots at flooding for the diluted catalyst and different gases. $\square$ air; $O$ argon; $\triangle$ helium; $\diamond$ Freon 12.

prudent policy to look for analogies with similar situations. For example it is known that the pressure drop $\Delta \mathbf{P}$ in a straight tube is proportional to the tube length $L$, to the energy content per unit volume of the fluid flowing through the tube $\frac{1}{2} \rho u_{\mathrm{g}}^{2}$ and to the surface area per unit volume of the fluid $4 / d_{t}$. This leads to the well-known relation $\Delta P d_{\mathrm{l}} / L \frac{1}{2} \rho_{\mathrm{g}} u_{\mathrm{g}}^{2}=4 f$. Here the friction factor $f$ is a function of the Reynolds number $\mathrm{Re}$, the ratio of inertia forces per unit of fluid volume $\frac{1}{2} \rho_{\mathrm{g}} u_{\mathrm{g}}^{2}$ to the viscous forces per unit of fluid volume $\eta_{g} u_{\mathrm{g}} / d_{\mathrm{t}}$, so $\operatorname{Re}=\rho_{\mathrm{g}} u_{\mathrm{g}} d_{\mathrm{t}} / \eta_{\mathrm{g}}$. In a packed bed with spheres the analogy results in $\Delta P$ being proportional to $L, \frac{1}{2} \rho_{\mathrm{g}}\left(u_{\mathrm{g}} / \varepsilon_{\mathrm{p}}\right)^{2}$ and the surface area per unit volume of the fluid $6\left(1-\varepsilon_{\mathrm{p}}\right) / \varepsilon_{\mathrm{p}} d \mathrm{p}$, where $\varepsilon_{\mathrm{p}}$ is the void fraction of the packed bed. This leads to $\triangle P \varepsilon_{\mathrm{p}} d_{\mathrm{p}}[12 L(1$ $\left.\left.-\varepsilon_{\mathrm{p}}\right) \rho_{\mathrm{g}}\left(u_{\mathrm{g}} / \varepsilon_{\mathrm{p}}\right)^{2}\right]=\mathcal{f}$, whereas $\mathrm{Re}$ is the ratio of $\rho\left(u_{\mathrm{g}} / \varepsilon_{\mathrm{p}}\right)^{2}$ to $\eta\left(u_{\mathrm{g}} / \varepsilon_{\mathrm{p}}\right) /\left[\varepsilon_{\mathrm{p}} d_{\mathrm{p}} /\left(1-\varepsilon_{\mathrm{p}}\right)\right]$ or $\operatorname{Re}_{\mathrm{pb}}$ $=\rho u_{\mathrm{g}} d \mathrm{p} / \eta\left(1-\varepsilon_{\mathrm{p}}\right)$. According to Ergun (1952) $f$ $=150 / \operatorname{Re}_{\mathrm{pb}}+1.75$. If non-spherical particles are used then $d_{\mathrm{p}}=6 \mathrm{~V} / S_{\mathrm{p}}$. If the surface area of the wall cannot be neglected compared to the particles surface area $\left(d_{1} / d_{\mathrm{p}}<10\right)$, then $d_{\mathrm{p}}=\frac{6\left(1-\varepsilon_{\mathrm{p}}\right)}{S_{\mathrm{v}}+4 / d_{\mathrm{t}}}$, where $S_{\mathrm{v}}$ is the external surface area of the packing per unit volume of the packed bed. The Ergun equation

$$
\frac{\Delta P \varepsilon_{\mathrm{p}} d_{\mathrm{p}}}{L\left(1-\varepsilon_{\mathrm{p}}\right) \rho_{\mathrm{g}}\left(u_{\mathrm{g}} / \varepsilon_{\mathrm{p}}\right)^{2}}=12 f=\frac{K_{\mathrm{L}}}{\operatorname{Re}_{\mathrm{pb}}}+K_{\mathrm{t}}
$$

where $K_{\mathrm{L}}=150$ and $K_{\mathrm{t}}=1.75$, can be checked for the various packing types that we have investigated.

Now the question can be raised which pressure drop can be expected if a solid trickles through a packed bed. For the countercurrent gas-solid trickle flow over a packed bed two distinct flow patterns have been observed; they are called the preloading regime and the loading regime. The operating point where the system passes from one regime to the other is called the loading point. 


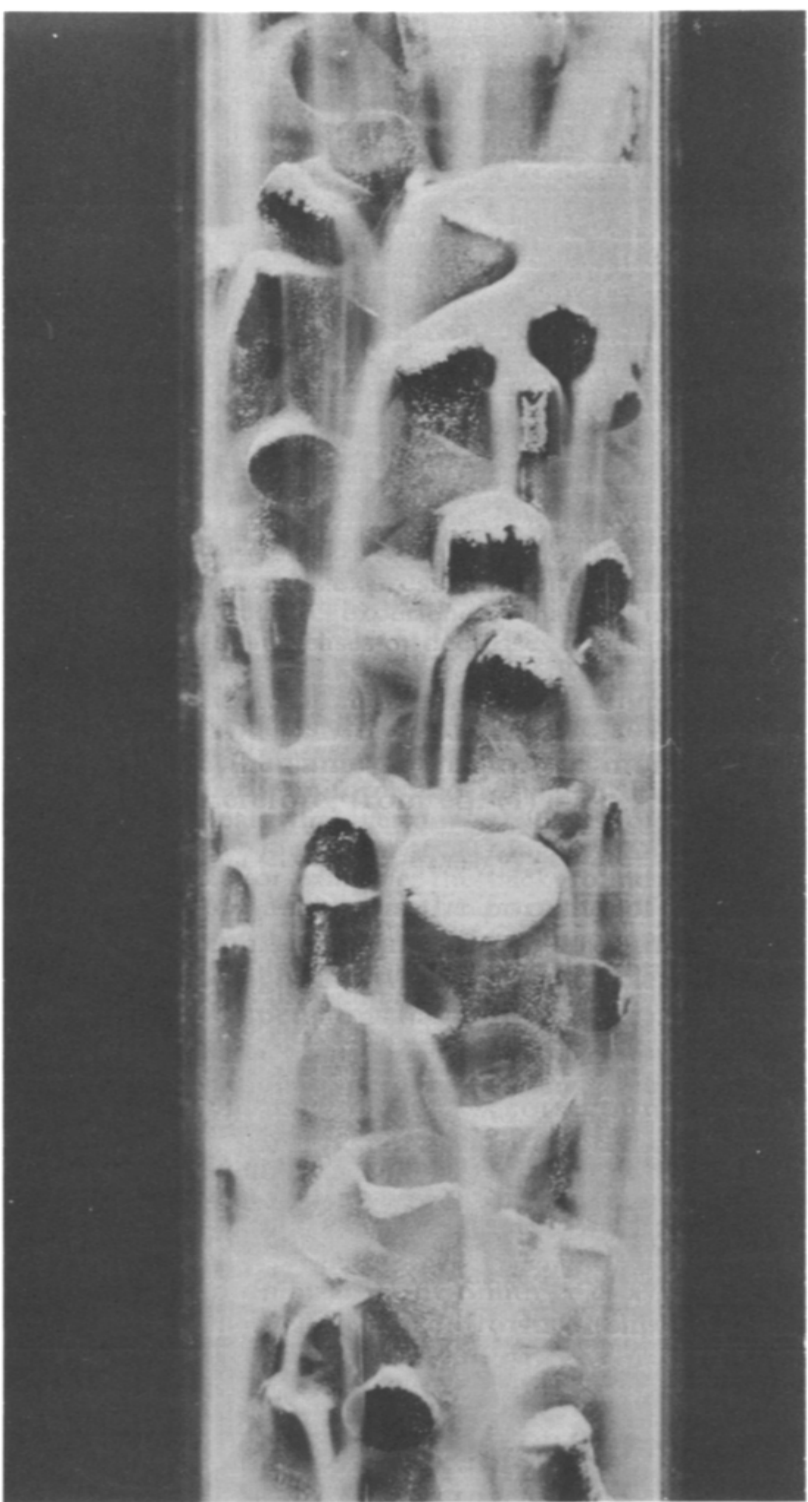

Plate 1. The trickle flow of a silica-alumina powder (average particle diameter $\bar{d}_{\mathrm{p}} \approx 90 \times 10^{-6} \mathrm{~m}$ ) over a dumped packing consisting of $5 \times 5 \mathrm{~mm}$ catalyst pellets and $7 \times 7 \times 1 \mathrm{~mm}$ glass Raschig rings. The ratio of the pellets to the rings equals 2 to 1 by number and the tube diameter is $25 \times 10^{-3} \mathrm{~m}$.

In the preloading zone the solid flows almost exclusively in the form of trickles: solids particles moving independently of these trickles are practically absent. The number of the solids trickles per unit of the cross-sectional area of the column has been observed to be independent of the gas and the solids flow rates. On the other hand, of course, the number of trickles will strongly depend on the geometry of the packing: the larger the number of interstices between the packing elements the more trickles can be formed. Roes and van Swaaij (1978) for the preloading zone have observed that the mean particle velocity $u_{\mathrm{p}}^{\circ}$, defined as

$$
u_{\mathrm{p}}^{\mathrm{o}}=\frac{S}{\rho_{\mathrm{p}} \beta_{\mathrm{dyn}}}
$$

is independent of the gas flow rate. Since both the number and the average velocity of the trickles do not depend on $S$, at increasing solids flow rates only the diameter of the solids trickles must increase.

Due to the flow of solids an increase of the pressure drop over the bed can be observed. We assume that gas is dragged along by the trickles flowing downwards. The proposed mechanism of such a gas-solid interaction is shown in Fig. 11. It has been observed that the trickles have a much higher void fraction than a fixed bed. The gas contained in between the particles moves downwards together with the trickle, is released partly on impingement on a catalyst particle, and flows upwards again. This results in an internal gas recirculation. The trickles on colliding with the fixed packing elements release the gas contained and now have a void fraction equal or only slightly higher than that of a fixed bed of the powder. After sliding down over the packing boundary the particle stream again starts falling by gravity: a new trickle is then formed, a new portion of the gas is dragged along and transported downwards until impingement on the next catalyst particle. The distance between two collisions varies according to our observations and equals approximately 2 to 5 times the geometric diameter of a packing element. We now can understand that $u_{\mathrm{p}}^{\mathrm{o}}$ is a velocity averaged between the velocity of a falling trickle and the velocity of the trickle sliding over the packing.

The trickling of the solids causes an increase of the pressure drop, because:

(1) the free cross-sectional area available for the gas flow is reduced by the trickles, increasing the superficial gas velocity;

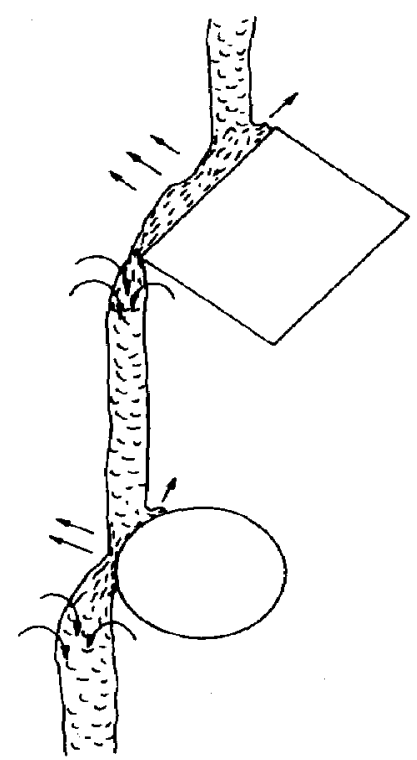

Fig. 11. The gas recirculation by a solids trickle. 
(2) the gas flow rate in upward direction inside the column is higher than the feed flow rate because of the internal recirculation of gas. This leads to an increase of the linear gas velocity.

So we assume that in principle the Ergun equation remains valid for the GSTF provided the real linear gas velocity in the packed column is taken into account.

We further assume in the trickles a certain void fraction $\varepsilon_{\mathrm{g}, \mathrm{tr}}$, defined as

$$
\varepsilon_{\mathrm{g}, \mathrm{tr}}=\frac{\text { gas volume in the trickle }}{\text { total trickle volume }}
$$

$\varepsilon_{\mathrm{g}, \text { tr }}$ is assumed to be constant and to be only characteristic for the system packing-trickling solid. The upward gas flow is increased by the amount dragged along with the solids, i.e. by $\frac{S}{\rho_{\mathrm{p}}} \frac{\varepsilon_{\mathrm{g}, \mathrm{tr}}}{1-\varepsilon_{\mathrm{g}, \mathrm{tr}}}$. As outlined in Fig. 12, the free cross-sectional area of the column available for the gas flow is reduced by the trickles, by the static hold-up and by the stagnant gas phase contained in between the particles of the static hold-up, that is by a factor of $\left(\varepsilon_{\mathrm{p}}-\frac{\beta_{\mathrm{dyn}}}{1-\varepsilon_{\mathrm{gtr}}}-\beta_{\mathrm{st}} \frac{\rho_{\mathrm{p}}}{\rho_{\mathrm{fb}}}\right)^{-7}$ where $\rho_{\mathrm{fb}}$ is the fixed bed density of the powder. So the real gas velocity $u_{\beta}$ as to be used in the Ergun equation (1) becomes:

$$
u_{\beta}=\frac{\frac{G}{\rho_{\mathrm{g}}}+\frac{S}{\rho_{\mathrm{p}}} \frac{\varepsilon_{\mathrm{e}, \mathrm{tr}}}{1-\varepsilon_{\mathrm{g}, \mathrm{tr}}}}{\left(\varepsilon_{\mathrm{p}}-\beta_{\mathrm{st}} \frac{\rho_{\mathrm{p}}}{\rho_{\mathrm{fb}}}-\frac{S}{\rho_{\mathrm{p}} u_{\mathrm{p}}^{\mathrm{o}}\left(1-\varepsilon_{\mathrm{g}, \mathrm{Ir}}\right)}\right)} .
$$

Using eq. (4), the pressure drop can be predicted if $\varepsilon_{\mathrm{g}, \mathrm{tr}}$ is known, or vice versa, $\varepsilon_{e, t r}$ can be determined from the experimental data on the pressure drop.

On increasing the gas or the solids flow rates the loading point will be reached. This is the upper limit of the preloading regime. Above this limit, a further increase of the gas flow rate leads to an increase of the dynamic holdup. In a $\beta_{\text {dyn }}$ vs $G$ plot, a line connecting the loading points for different $S$-values is called the "loading line".

On a microscale we observe that at and beyond the loading point freely moving particles appear. The trickle flow is now accompanied by a dynamic interaction between particles and the gas surrounding the trickles. Particles located near the surface of a trickle can easily be torn off from this trickle, they then move suspended freely in the gas until they are snatched again by a trickle. The pressure drop in the column is now increased by the weight of the freely suspended particles. In the preloading region the fraction of $\beta_{\text {dyn }}$ which is suspended is assumed to be negligible. If possibly in the preloading regime a particle becomes released from a trickle, it should return quickly to the trickle. Therefore it must be able to move in the downward direction: its terminal velocity $u_{t}$ must be higher than the real gas velocity in the column. At the loading point these two velocities become equal, so that for a given packing and a given gas-solid system holds

$$
u_{\mathrm{t}}=u_{\mathrm{LP}}=u_{\beta, \text { at start of loading }}
$$

or also $\mathrm{d} u_{\beta}=0$. We have to realise that $u_{\mathrm{t}}$ relates to a particle diameter somewhere in the lower range of the particle size distribution of the powder used.

Using eq. (4) and assuming that $\varepsilon_{\mathrm{g}, \mathrm{tr}}$ is a constant, we can write for the loading line at low values of $S$ (so that

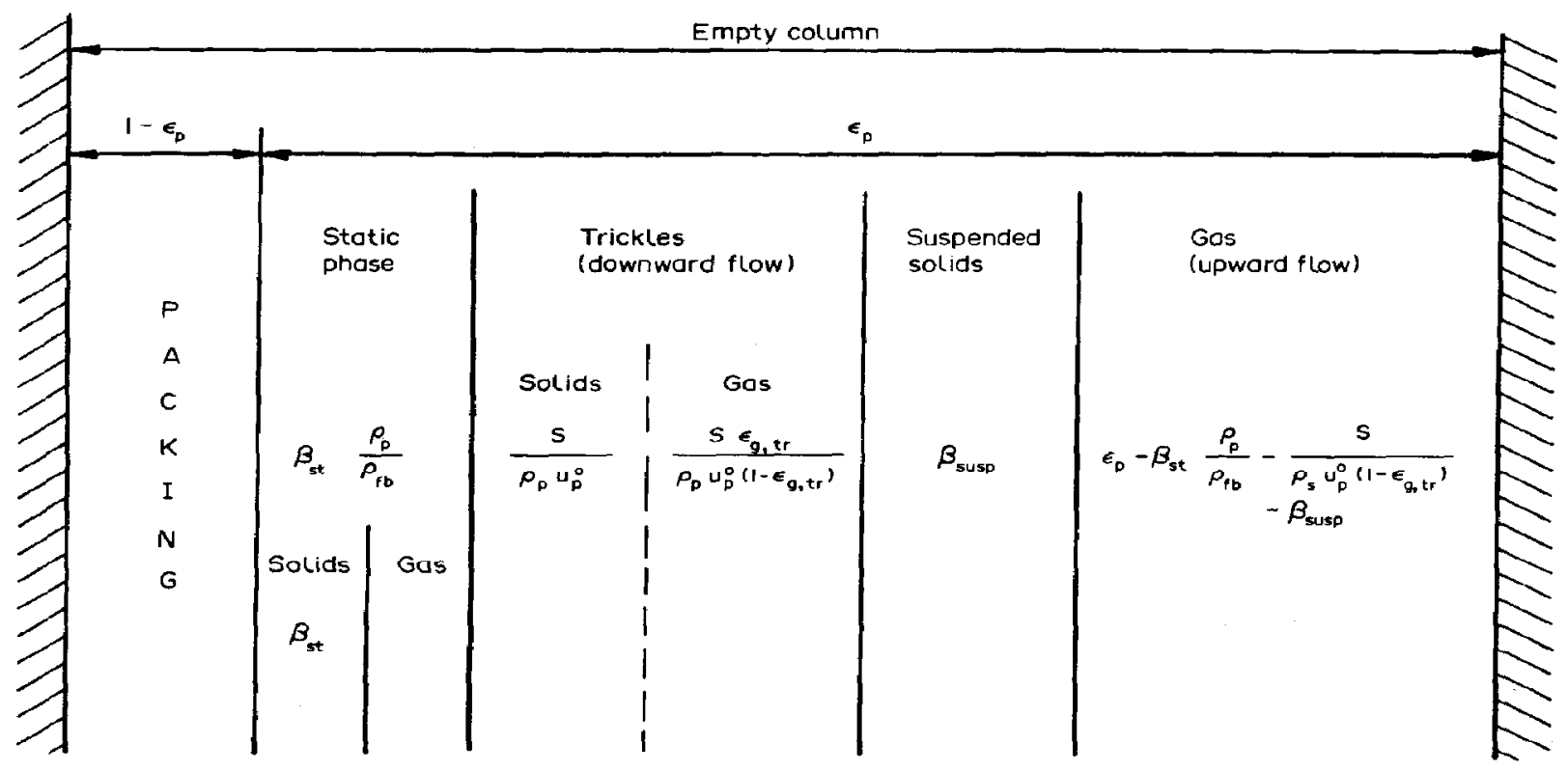

Fig. 12. Stationary and mobile phases in the GSTF column. 
the denominator of eq. (4) can be considered as a constant):

$$
\begin{aligned}
\mathrm{d} u_{\beta}=0=\frac{1}{\rho_{\mathrm{g}}} \mathrm{d} G+\frac{\varepsilon_{\mathrm{g} . \mathrm{tr}}}{\rho_{\mathrm{p}}\left(1-\varepsilon_{\mathrm{g}, \mathrm{tr}}\right)} \mathrm{d} S \text { or } \\
\quad\left(\frac{\mathrm{d} S}{\mathrm{~d} G}\right)_{\text {loading }}=-\frac{\rho_{\mathrm{p}}}{\rho_{\mathrm{g}}} \frac{\left(1-\varepsilon_{\mathrm{g}, \mathrm{tr}}\right)}{\varepsilon_{\mathrm{g}, \mathrm{tr}}} .
\end{aligned}
$$

So, $\varepsilon_{g, t r}$ can be determined from the slopes of the loading lines which are expected to be straight lines.

The steady-state motion of a single particle is described by the Stokes' law:

$$
u_{\mathrm{t}}=\frac{g\left(\rho_{\mathrm{p}}-\rho_{\mathrm{g}}\right) d_{\mathrm{part}}^{2}}{18 \rho_{\mathrm{g}} v_{\mathrm{g}}}
$$

for $\operatorname{Re}_{\text {part }}=\frac{u_{\mathrm{t}} d_{\text {part }}}{v_{\mathrm{g}}}<2$ (McCabe and Smith, 1976). In dimensionless terms eq. (7) can be rewritten (Wesselingh, 1986) into:

$$
u^{*}=\frac{1}{18}\left(d^{*}\right)^{2}
$$

where $u^{*}=\frac{u_{\mathrm{t}}}{u_{\mathrm{r}}}$ and $d^{*}=\frac{d_{\mathrm{part}}}{d_{\mathrm{r}}}$ are the dimensionless terminal velocity and particle diameter respectively. The reference velocity and reference diameter are defined by $u_{\mathrm{r}}=\left(g^{\prime} v_{\mathrm{g}}\right)^{1 / 3}$ and $d_{\mathrm{r}}=\left(v_{\mathrm{g}}^{2} / g^{\prime}\right)^{1 / 3}$ with $g^{\prime}=g\left(\rho_{\mathrm{p}}-\rho_{\mathrm{g}}\right) / \rho_{\mathrm{g}}$.

At the loading point, the upward gas velocity equals the terminal velocity of the solids particles. For a given packing and a given powder with a given distribution of particle diameters, a logarithmic plot of $u^{*}$ versus $\left(\frac{1}{d_{r}}\right)$ therefore is expected to be a straight line.

In the loading region we assume that the trickles no more change in size, because the amount of solids fed at the top of the column under steady state conditions also has to leave at the bottom. So the net downflowing stream of solids is constant. Also a steady state is reached in the amount of solids particles torn off from the trickles and the amount of suspended particles snatched again by the trickles. Now the dynamic holdup in the packed bed consists of two contributions: the constant content of the trickles $\beta_{\text {tr }}$ and the freely suspended $\beta_{\text {susp. }}$. So for $\beta_{\mathrm{dyn}}$ we have

$$
\beta_{\mathrm{dyn}}=\beta_{\mathrm{tr}}+\beta_{\text {susp }}=\frac{S}{\rho_{\mathrm{p}} u_{\mathrm{p}}^{\circ}}+\beta_{\text {susp }}
$$

At constant gas flow rate $G$ the real gas velocity $u_{\beta}$ now is increased due to the suspended particles, so that eq. (4) has to be rewritten as:

$$
u_{p}=\frac{\frac{G}{\rho_{\mathrm{g}}}+\frac{S}{\rho_{\mathrm{p}}} \frac{\varepsilon_{\mathrm{g}, \mathrm{tr}}}{1-\varepsilon_{\mathrm{g}, \mathrm{tr}}}}{\left(\varepsilon_{\mathrm{p}}-\beta_{\mathrm{st}} \frac{\rho_{\mathrm{p}}}{\rho_{\mathrm{fb}}}-\frac{S}{\rho_{\mathrm{p}} u_{\mathrm{p}}^{\mathrm{o}}\left(1-\varepsilon_{\mathrm{g}, \mathrm{tr}}\right)}-\beta_{\text {susp }}\right)} .
$$

Flooding is the upper limit of the loading zone and can be caused by either of two phenomena (van Swaaij and Verver, 1986): solids blow-off or clogging. If there is no recycling of the entrained solids back to the top of the column, at a sufficient gas flow the lighter particles are blown out of the column and only the heavier particles move downwards. Here the hold-up passes through a maximum. At a further increase of gas flow ratc the hold-up decreases. If the entrained solids particles are recirculated back into the column, e.g. by means of a cyclone, both the hold-up and the pressure drop increase very quickly and the column becomes completely clogged. In the flooding regime the operation of a GSTF-column is unstable, so quantitative relations between the hold-up and the flow rates cannot be formulated.

\section{CORRELATION OF THE RESULTS}

To determine the particle velocity $u_{\mathrm{p}}^{\circ}$ we plot the dynamic holdup versus the solids flow rate in the preloading region, as is shown in Fig. 13. For the diluted catalyst (Fig. 13a) the points lie exactly on a straight line for all four gases used. This confirms the validity of eq. (2). For the diluted catalyst we find $u_{\mathrm{pDC}}^{\mathrm{o}}$ $=0.12 \mathrm{~m} / \mathrm{s}$ and for the Raschig rings (Fig. 13b) $u_{\mathrm{pRR}}^{\mathrm{o}}=0.17 \mathrm{~m} / \mathrm{s} . u_{\mathrm{p}}^{\mathrm{o}}$ appears to be independent of the properties of the gas phase; it only depends on the geometry of the packing and perhaps also on the properties of the powder, which have not been varied by us.

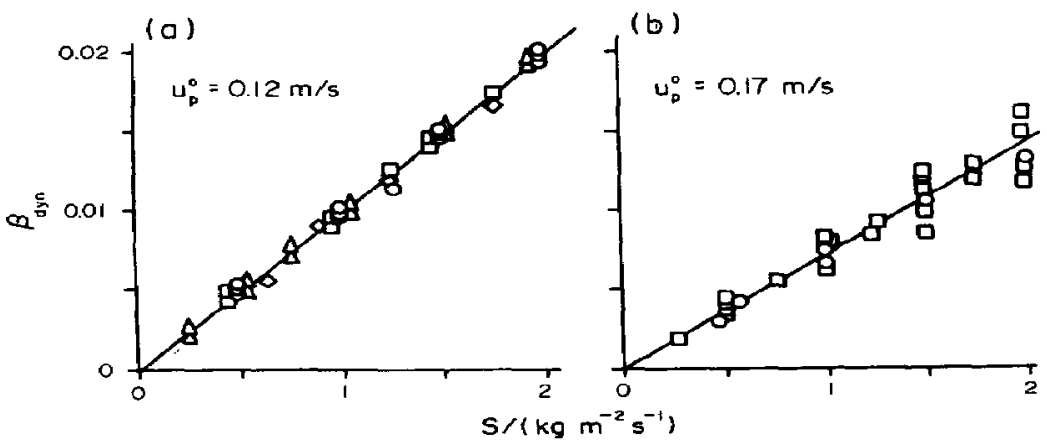

Fig. 13. The dynamic holdup in the preloading zone versus the solids flow rate for (a) the diluted catalyst and (b) the Raschig rings. For symbols see Fig. 10. 
For Kerapak the trickle velocity is not constant: it decreascs at increasing gas flow rates, cven at low gas velocities. Most probably this different behaviour is caused by the regular geometry of this packing with no vertical channels. In the regularly arranged, sloped channels the trickles slide down along the walls, whereas freely falling trickles are almost absent. So an internal recirculation of gas will hardly take place.

The pressure drop without trickle flow experiments for the three packings have been correlated using the Ergun equation (1). In our case the ratio of the column to the packing diameter is 3 to 5 and therefore we introduced the usual apparent particle diameter according to $d_{\mathrm{p}}=\frac{6\left(1-\varepsilon_{\mathrm{p}}\right)}{S_{\mathrm{v}}+\frac{4}{d_{\mathrm{t}}}}$. From a plot of $\frac{\varepsilon_{\mathrm{p}}^{3} \Delta P d_{\mathrm{p}}}{\left(1-\varepsilon_{\mathrm{p}}\right) L \rho_{\mathrm{g}} u_{\mathrm{g}}^{2}}$ versus $\operatorname{Re}^{-1}=\left[\frac{u_{\mathrm{g}} d_{\mathrm{p}}}{v_{\mathrm{g}}\left(1-\varepsilon_{\mathrm{p}}\right)}\right]^{-1}$, both $K_{\mathrm{L}}$ and $K_{\text {, }}$ can be determined. The values obtained for each individual gas are given in Table 5. For the diluted catalyst the average values for all the investigated gases together are 140 and 1.52 , respectively. These values are slightly lower than those of 150 and 1.75 as originally proposed by Ergun. For both the Raschig rings and the Kerapak the calculated $K_{L}$ and $K_{1}$ values are significantly lower than the Ergun's values as can be seen in the table. We should realise that the condition $d_{t} / d_{p}>20$ for the validity of Ergun's correlation does not hold in our case.

In Figure 14 we compare our experimental data for the diluted catalyst with the Ergun correlation. Our experimental points are within the accuracy found by Ergun for his correlation. However, for the individual gases we can observe a systematic deviation between the correlation and the experiments. We have no explanation for these deviations. For Raschig rings the experimental points lie approximately $50 \%$ below the Ergun curve. We think it is doubtful whether the usual method to determine an equivalent particle diameter is valid for shapes which deviate so strongly from a sphere as for example in the case of a hollow cylinder.

From the experimental data on the pressure drop with trickle flow in the preloading zone, the void fraction $\varepsilon_{\mathrm{g} \text {, tr }}$ of the trickles can be calculated by a search for the value of $\varepsilon_{\text {,tr }}$, which gives the best fit of the Ergun equation using the gas velocity $u_{\beta}$ as given by eq. (4). For the diluted catalyst and the four gases used we find for $\varepsilon_{\text {g.tr }}$ an average value of 0.936 . For the Raschig rings and air $\varepsilon_{\text {g.tr }}=0.934$. In Figures 2(a), (b) and 3 the calculated lines are shown together with the experimental points. Within the experimental accuracy $\varepsilon_{\mathrm{g}, \mathrm{tr}}$ appears to be independent of the properties of the gas phase. In the loading region the measured values of

Table 5. The parameters $K_{\mathrm{L}}$ and $K_{\mathrm{t}}$ as calculated from the experimental data

\begin{tabular}{|c|c|c|c|c|c|}
\hline Packing & gas & argon & air & helium & Freon 12 \\
\hline $\begin{array}{l}\text { diluted } \\
\text { catalyst }\end{array}$ & $\begin{array}{l}\operatorname{Re}_{\mathrm{pb}} \\
K_{\mathrm{L}} \\
K_{\mathrm{t}}\end{array}$ & $\begin{array}{c}6.5-89 \\
127 \\
1.35\end{array}$ & $\begin{array}{l}c^{4.9-110} \\
1.98\end{array}$ & $\begin{array}{l}0.95-38 \\
96 \\
4.04\end{array}$ & $\begin{array}{l}25-750 \\
223 \\
1.32\end{array}$ \\
\hline $\begin{array}{l}\text { Raschig } \\
\text { rings }\end{array}$ & $\begin{array}{l}\operatorname{Re}_{p b} \\
K_{L} \\
K_{1}\end{array}$ & $\begin{array}{l}6.2-110 \\
100 \\
0.99\end{array}$ & $\begin{array}{l}9.8-190 \\
78 \\
0.97\end{array}$ & & \\
\hline Kerapak & $\begin{array}{l}\operatorname{Re}_{p b} \\
K_{L} \\
K_{t}\end{array}$ & & $\begin{array}{l}9.8-77 \\
70 \\
0.43\end{array}$ & & \\
\hline
\end{tabular}

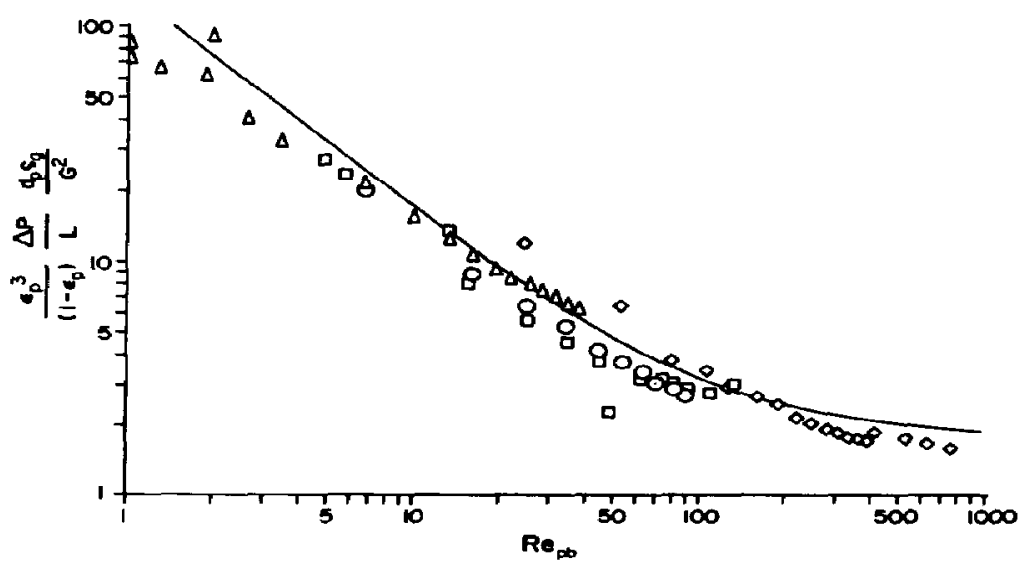

Fig. 14. The experimental pressure gradient data in an Ergun plot. For symbols see Fig. 10; $-=$ Ergun. 
$\Delta P / L$ are higher than those predicted: this excessive increase of the pressure drop is caused by the weight of the suspended solid particles.

The loading lines obtained from $\beta_{\text {st }}-G$ plots are approximately straight lines, see Fig. 6. Using eq. (6) and taking for $\varepsilon_{\mathrm{g}, \mathrm{r}}$ the value obtained from the pressure drop data, we can predict the slopes of the loading lines. The lines $P-P^{\prime}$ in Fig. 6(a) and (b) have slopes calculated from eq. (6). Within the accuracy of our $\beta_{\text {dyo }}$ vs $G$ plots these lines properly predict the locations of the loading points.

In Fig. 15 for constant values of $S$ we have plotted $u_{\mathrm{LP}}^{*}=\frac{u_{\mathrm{LP}}}{u_{\mathrm{r}}}$ versus $d^{*}=\frac{d_{\text {part }}}{d_{\mathrm{r}}}$ for the diluted catalyst. $u_{L P}$ is the real velocity of the gas at the loading point, that is the corresponding value of $u_{\beta}$. Here for $d_{\text {part }}$ we took a value of $70 \times 10^{-6} \mathrm{~m}$ : for the powder used by us we expect that the loading starts when particles having approximately this diameter become unable to fall down. The data in Fig. 15 can be correlated by:

$$
u_{\mathrm{LP}}^{*}=2.3\left(d^{*}\right)^{1.3}\left(\frac{S}{\rho_{\mathrm{p}} u_{\mathrm{p}}^{\circ}}\right)^{0.5}
$$

The following can be said of this correlation. Our operating region lies around the boundary of the validity of the correlations $u^{*}=\left(d^{*}\right)^{2} / 18$ to $u^{*}=$ $0.23 d^{*}$ for the velocity of a freely falling particle (Wesselingh, 1986). We see in our correlation that the

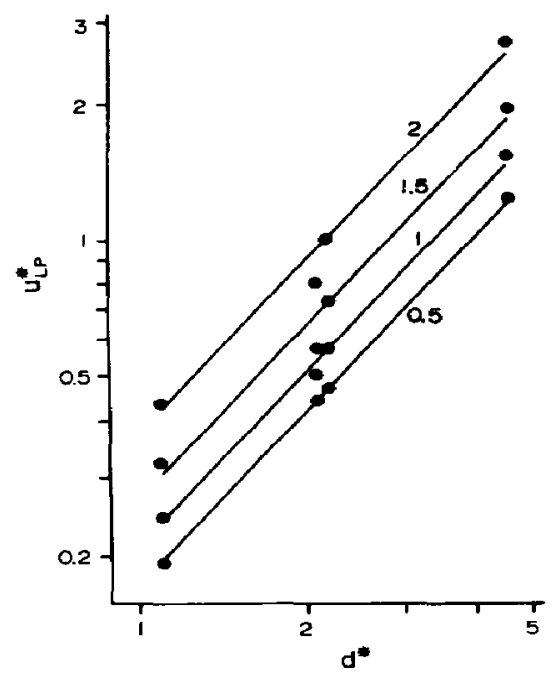

Fig. 15. $u_{\mathrm{L}^{*}}^{*}$ as a function of $d^{*}$. Parameter is the solids flow rate $S\left(\mathrm{~kg} \mathrm{~m}^{-2} \mathrm{~s}^{-1}\right)$ exponent lies between 2 and 1 . The $u_{\mathrm{LP}}^{*}$ is higher than the $u^{*}$ for a freely falling particle. This could be expected because of the protective character of the trickles: higher gas velocities are necessary to tear off the particles from the trickles. Further the value of $u_{L P}$ depends on $\sqrt{S}$. We further know, that the influence of the gas properties have been fully accounted for by introducing $d_{\mathrm{r}}$ and $u_{\mathrm{r}}$. So the effect of the solids flow rate only can be accounted for by introducing packing or solid powder properties. Arbitrarily we have taken $\rho_{\mathrm{p}} u_{\mathrm{p}}^{\circ}$, we have no proof for that. In future experiments we will try to elucidate this aspect.

In the loading range the slip velocity $u_{\text {slip }}$ as calculated according to Roes and Van Swaaij (1978) by

$$
u_{\text {slip }}=\frac{S}{\rho_{\mathrm{s}} \beta_{\mathrm{dyn}}}+\frac{G}{\rho_{\mathrm{g}}\left(\varepsilon_{\mathrm{p}}-\beta_{\mathrm{st}} \frac{\rho_{\mathrm{p}}}{\rho_{\mathrm{fb}}}-\beta_{\mathrm{dyn}}\right)}
$$

appears, within the accuracy of the data, to be independent of $S$ and $G$ in the loading range. The calculated values of $u_{\text {slip }}$ are given in Table 6 . For the Raschig rings we find a mean value of $u_{\text {slip }}=0.61 \mathrm{~m} / \mathrm{s}$, which is considerably higher than that for the diluted catalyst. So in contradiction to the expectations of Roes and Van Swaaij (1978), $u_{\text {slip }}$ strongly depends on the packing geometry and on the properties of the gas phase: for the diluted catalyst we find for argon, air and Freon $12 u_{\text {slip }} \approx 0.25 \mathrm{~m} / \mathrm{s}$ while for helium $u_{\text {slip }}=0.45$ $\mathrm{m} / \mathrm{s}$. We have no explanation for this difference.

\section{CONCLUSIONS}

The suitability of three different packing materials for gas-solid trickle flow has been evaluated by pressure drop and hold-up measurements. The ceramic Sulzer packing exhibits a low pressure drop and a good stability. No loading or flooding phenomena are observed for this packing. The region of stable operation is large, the static hold-up is low.

For dumped packings a pressure drop correlation is derived taking an internal gas recirculation into account. The concept of the "trickle void fraction" $\varepsilon_{\mathrm{x}, \mathrm{tr}}$ is introduced and substantiated. From the experiments follows, that a solid particle in a trickle drags along about 15 times its own volume as gas in the trickle. From the pressure drop measurements with various gases $\varepsilon_{\mathrm{g}, \mathrm{tr}}$ is found to be independent of the properties of the gas phase. In the preloading region the mean particle velocity is constant and independent of the properties of the gas phase and of the gas flow rate, so $\beta_{\text {dyn }}$ in this region can easily be predicted with eq. (2) if $u_{\mathrm{p}}^{\circ}$ is known. For the diluted catalyst $u_{\mathrm{p}}^{\circ}=0.12 \mathrm{~m} / \mathrm{s}$ and

Table 6. The mean values of $u_{\text {slip }}$ calculated with eq. (12)

\begin{tabular}{lllll}
\hline Packing & argon & air & helium & Freon 12 \\
\hline & & $u_{\text {stip }}(\mathrm{m} / \mathrm{s})$ & & \\
& & 0.24 & 0.45 & 0.25 \\
diluted catalyst & 0.26 & 0.61 & & \\
Raschig rings & & & & \\
\hline
\end{tabular}


for the Raschig rings $u_{\mathrm{p}}^{\circ}=0.17 \mathrm{~m} / \mathrm{s}$. The pressure drop in the preloading region can be predicted reliably by using the linear gas velocity according to eq. (4) in the Ergun equation (1). For the trickle void fraction $\varepsilon_{\text {g.r }}$ we have found the value of $0.93^{5}$. The preloading regime ends as soon as $u_{\beta}=u_{\text {L.P. }}$. The value of $u_{\text {LP }}$ can be calculated for the diluted catalyst with eq. (11).

From our experimental data we are unable to derive a universal relation allowing a reliable prediction of the dynamic hold-up in the loading range and of flooding. More experimental data are necessary especially in the operating region near flooding. For a rough estimation of $\beta_{\mathrm{dyn}}$ in the loading regime the use of eq. (12) may be recommended, using for $u_{\text {slip }}$ the values reported for the different gases.

Acknowledgements-The authors thank the Twente University of Technology and the Royal Dutch Shell Group for the financial support and $M r$. G. Poort and $M r$. W. Smeitink for their contribution to the experimental work.

\section{NOTATION}

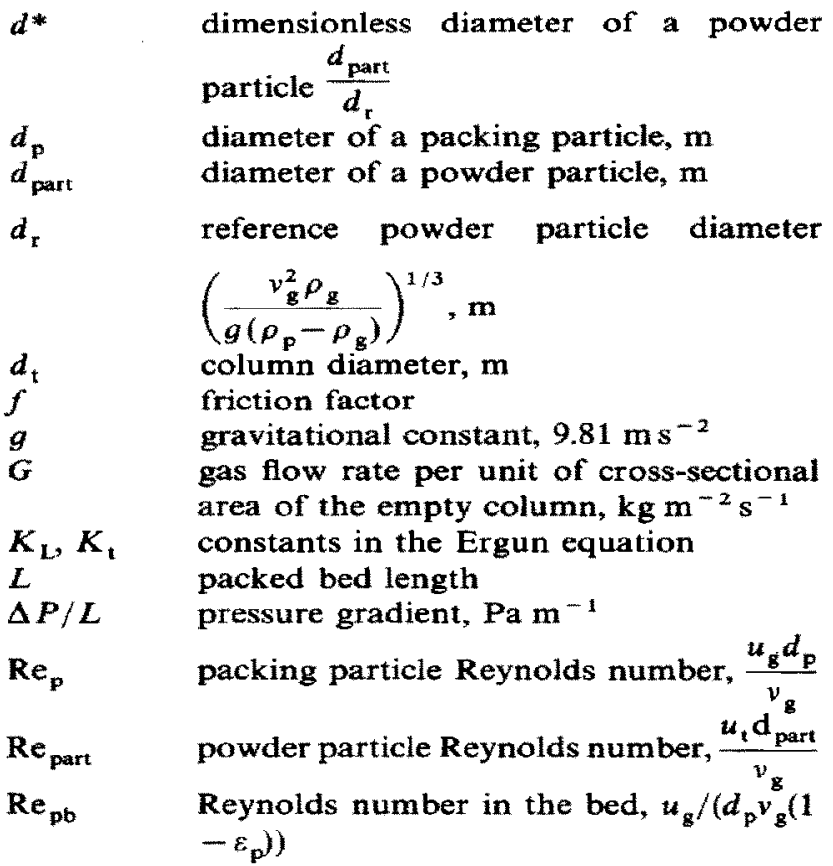

$S_{\mathrm{p}}$

$S_{\mathbf{v}}$

$u^{*}$

$u_{\mathrm{g}}$

$u_{\mathrm{LP}}$

$u_{\mathrm{p}}^{\mathrm{o}}$

$u_{t}$

$u_{r}$

$u_{\text {slip }}$

$V_{p}$

$\beta_{\text {dyn }}^{p}$

$\beta_{\text {si }}$

$\beta_{\text {tr }}$

$E_{\text {git }}$

$\beta_{\text {susp }}$

$\varepsilon_{p}$

$P \mathrm{~b}$

$\rho_{\mathrm{g}}$

$\rho_{p}$

$v$ solids flow rate per unit of cross-sectional area of cmpty column, $\mathrm{kg} \mathrm{m}^{-2} \mathrm{~s}^{-1}$

external surface area of single packing particle, $\mathrm{m}^{2}$

surface area of the packing per unit bed volume, $\mathrm{m}^{-1}$

dimensionless terminal velocity, $\frac{u_{t}}{u_{\mathrm{r}}}$ approach velocity of the gas, $\mathrm{m} \mathrm{s}^{-1}$ real gas velocity at the loading point, $\mathrm{m} \mathrm{s}^{-1}$

particle velocity in the preloading region, $\mathrm{ms} \mathrm{s}^{-1}$

terminal particle velocity, $\mathrm{ms}^{-1}$ reference terminal velocity,

$$
\left(\frac{g\left(\rho_{\mathrm{p}}-\rho_{\mathrm{g}}\right) v_{\mathrm{g}}}{\rho_{\mathrm{g}}}\right)^{1 / 3}, \mathrm{~ms}^{-1}
$$

slip velocity (eq. (12)), $\mathrm{ms}^{-1}$

volume of a single packing particle, $\mathrm{m}^{3}$ dynamic holdup of the solid powder static holdup of the solid powder dynamic holdup contained in trickles void fraction of a trickle suspended fraction of dynamic holdup void fraction of the packing

fixed bed density, $\mathrm{kg} \mathrm{m}^{-3}$

gas density, $\mathrm{kg} \mathrm{m}^{-3}$

density of the powder particle, $\mathrm{kg} \mathrm{m}^{-3}$ kinematic viscosity, $\mathrm{m}^{2} \mathrm{~s}^{-1}$

\section{REFERENCES}

Claus, G., Vergnes, F. and Le Goff, P., 1976, Hydrodynamic study of gas and solid flow through a screen-packing. Can. $J$. chem. Engng 54, 143-147.

Ergun, S. 1952, Fluid flow through packed columns. Chem. Engng Progr. 48, 8994.

Large, J. F., Naud, M., Guigon, P. and Bergougnou, M. A., 1981 , Hydrodynamics of the raining packed-bed gas-solids heat exchanger. Chem. Engng J. 22, 95-100.

McCabe, W. L. and Smith, J. C., 1976, Unit Operations of Chemical Engineering. McGraw-Hill, New York, p. 154.

Roes, A. W. M. and van Swaaij, W. P. M., 1978, Hydrodynamic behaviour of a gas-solid countercurrent packed column at trickle flow. Chem. Engng $J$. 17, 81-89.

van Swaaij, W. P. M. and Verver, A. B., Gas-solids trickle flow. In: Proc. 16th ICHMT Symposium Heat and Mass Transfer in Fixed and Fluidized Beds, Dubrovnik, Sept. 3-7, 1984, Springer, Berlin, pp. 1-26.

Wesselingh, J. A., 1987, The velocity of particles, drops and bubbles. Chem. Engng Process 21, 9-14. 